\title{
MicroRNA-564 inhibits the progression of non-small cell lung cancer via targeting plexin A4
}

\author{
HUI DING ${ }^{1}$, LIANGPENG LI ${ }^{2}$, BIAO GU $^{1}$, YAOJUN NI ${ }^{1}$ and SHENG CHEN ${ }^{1}$ \\ ${ }^{1}$ Department of Thoracic Surgery, Huai'an First People's Hospital, \\ The Affiliated Huaian No. 1 People's Hospital of Nanjing Medical University, Huai'an, Jiangsu 223300; \\ ${ }^{2}$ Department of Thoracic Surgery, Nanjing First Hospital, Nanjing Medical University, P.R. China
}

Received March 28, 2020; Accepted December 9, 2020

DOI: $10.3892 /$ etm.2021.9789

\begin{abstract}
Non-small cell lung cancer (NSCLC) is the most frequently diagnosed type of cancer, and the most prevalent cause of cancer-associated mortality. The present study aimed to investigate whether microRNA (miR)-564 influences NSCLC progression by regulating NSCLC cell growth and migration, via targeting plexin A4. Therefore, the expression levels of miR-564 and plexin A4 were evaluated in NSCLC specimens or cells using reverse transcription-quantitative PCR. Furthermore, colony formation and Cell Counting Kit-8 assays were performed to determine the proliferative ability of NSCLC cells. The cell migration capacity was assessed using a Transwell assay. In addition, to examine the binding ability of miR-564 on the plexin A4 3'-untranslated region (3'UTR), a dual-luciferase reporter assay was performed. A mouse xenograft model was established to evaluate the effect of miR-564 knockdown on tumor growth in vivo, whereas the expression of plexin A4 and Ki67 in NSCLC tissues was detected using immunohistochemistry. Notably, miR-564 was downregulated in both NSCLC cell lines and tissues, while its overexpression, following transfection with miR-564 mimics, attenuated the proliferation and proliferation, migration and invasion of NSCLC cells. By contrast, silencing of miR-564 using a miR-564 inhibitor promoted NSCLC cell proliferation, migration and invasion. The luciferase assay revealed that miR-564 directly targeted the plexin A4 3'UTR in A549 and H460 cells. Additionally, the overexpression of plexin A4 rescued the effect of miR-564 on NSCLC cell proliferation, migration and invasion abilities. Further in vivo studies demonstrated that miR-564 knockdown promoted
\end{abstract}

Correspondence to: Dr Sheng Chen, Department of Thoracic Surgery, Huai'an First People's Hospital, The Affiliated Huaian No. 1 People's Hospital of Nanjing Medical University, 6 Beijing West Road, Huai'an, Jiangsu 223300, P.R. China

E-mail: chenshengnj@outlook.com

Key words: simvastatin, elderly patients, carotid atherosclerotic plaque, serum inflammatory factors, cardiocerebrovascular events, microemboli
NSCLC growth, while miR-564 overexpression resulted in the opposite effect in nude mice. Overall, the results of the present study revealed that miR-564 promotes the proliferation and migration of NSCLC cells, both in vitro and in vivo, via targeting plexin A4. Therefore, miR-564 may be considered as a possible therapeutic target for NSCLC.

\section{Introduction}

Lung cancer is one of the most common causes of death, accounting for $\sim 26$ and $28 \%$ of all female and male cancer-related deaths, respectively, in 2013. Non-small cell lung cancer (NSCLC) accounts for $>80 \%$ of all lung cancer cases, and tops the list of the most commonly diagnosed type of cancer (1). Although great progress has been achieved in NSCLC therapy, the 5-year overall survival rate has remained unchanged at $<16 \%$ (2). Due to the poor prognosis, the development of novel strategies for the diagnosis and therapy of NSCLC is urgently required.

MicroRNAs (miRNAs/miRs) are single-stranded, small non-coding RNAs of 18-22 nucleotides in length, which can modulate gene expression. miRNAs bind to target mRNAs to regulate their expression via blocking the transcription or promoting transcriptional degradation (3). In addition, they participate in several biological processes, including cell proliferation, migration and apoptosis (4), and are involved in numerous types of cancer, such as gastric (5), breast (6) and lung cancer (7). miR-564 is a novel miRNA that has not been widely studied. Previous studies have reported that miR-564 is downregulated in prostate cancer, hepatocellular carcinoma, osteosarcoma, gastric carcinoma and glioblastoma (8-12). Furthermore, miR-564 acts as a tumor suppressor in the aforementioned types of cancers via targeting various mRNAs. MiRs may act as oncogenes or anti-cancer genes in different type of cancers. So far, the function of miR-564 in the development of NSCLC remains to be clarified. Moreover, the mechanism of miR-564 in regulating the cancer progression is limited. The present study tried to elucidate the presice mechanism of miR-564 in the development of NSCLC.

Plexin A4, a member of Plexin families, is the receptor of Semaphorins (Semas), which function as axonal guidance in nervous system (13). Plexin A4 modulates cell adhesion and migration via interacting with Sema6B, which is a 
promoter of tumorigenesis, immune responses, and tissue remodeling (14-16). Moroever, overexpression of Plexin A4 contributes to the inactivation of $\mathrm{T}$ cell (17). Depletion of Plexin A4 in $\mathrm{T}$ cells facilitates proliferative ability in vivo and in vitro. A previous study reveal that Plexin-A4 enhances tumor progression and angiogenesis (18). However, the potential roles of Plexin A4 in NSCLC has not been elucidated.

The current study demonstrated that miR-564 was downregulated in NSCLC cell lines and tissues. The effects of miR-564 on NSCLC cell proliferation and progression were also investigated. Herein, the human NSCLC cell lines A594 and H460 were utilized, whereas the effect of miR-564 on tumor growth in vivo was investigated in a nude mice xenograft model. Finally, bioinformatics analysis and luciferase reporter assays revealed that plexin A4 was directly targeted by miR-564 in NSCLC cells.

\section{Materials and methods}

Clinical tissues collection. Human NSCLC tumor and adjacent normal tissues were collected from 25 patients with NSCLC at the Huaian First People's Hospital. The tissues were isolated and immediately stored in liquid nitrogen. Patients signed informed consent in order for their specimens to be used for scientific research. The current study was approved by the Ethics Committee of the Huaian First People's Hospital.

Cell culture. The human bronchial epithelial (HBE) cell line and four NSCLC cell lines, namely A549, H1299, PC-9 and H460, were purchased from the American Type Culture Collection. Cells were cultured in DMEM (Gibco; Thermo Fisher Scientific, Inc.) supplemented with streptomycin (100 $\mu \mathrm{g} / \mathrm{ml})$, penicillin $(100 \mathrm{U} / \mathrm{ml})$ and 10\% FBS (Gibco; Thermo Fisher Scientific, Inc.), and incubated at $37^{\circ} \mathrm{C}$ in a humidified $5 \% \mathrm{CO}_{2}$ incubator.

Reverse transcription-quantitative PCR (RT- $q P C R)$. Total RNA was extracted from NSCLC specimens and cell lines using TRIzol reagent (Invitrogen; Thermo Fisher Scientific, Inc.), according to the manufacturer's instructions. A total of $1 \mu \mathrm{g}$ RNA was reverse transcribed into cDNA using the PrimeScript RT reagent kit (Takara Biotechnology Co., Ltd.). Furthermore, qPCR was carried out using the SYBR Premix Ex Taq kit (Beyotime Institute of Biotechnology) according to the manufacturer's instructions, on a FAST7500 Real-Time PCR system (Applied Biosystems; Thermo Fisher Scientific, Inc.). The PCR was performed under the following thermocyling conditions: $95^{\circ} \mathrm{C}$ for $30 \mathrm{sec}, 40$ cycles of $95^{\circ} \mathrm{C}$ for $5 \mathrm{sec}$ and $60^{\circ} \mathrm{C}$ for $34 \mathrm{sec}$. U6 and GAPDH served as loading control for miRNA and mRNA, respectively. The results were calculated by $2^{-\Delta \Delta \mathrm{Cq}}$ method (19).

Cell transfection. miR-564 mimics, inhibitor and the corresponding negative controls were obtained from Shanghai GenePharma Co., Ltd. The construction of the plexin A4 overexpression plasmid (pcDNA3.1/plexin A4) was also completed by Shanghai GenePharma Co., Ltd. The miR-564 antagomiR and negative control were purchased from Guangzhou RiboBio Co., Ltd. Cells were transfected using Lipofectamine 3000 reagent (Invitrogen; Thermo Fisher Scientific, Inc.) according to the manufacturer's protocol.
Cell viability assay. Subsequently, $1 \times 10^{3}$ A549 or H460 cells were seeded into 96 -well plates $24 \mathrm{~h}$ post-transfection. Following 12, 24, 48 and $72 \mathrm{~h}$ of incubation, the medium was replaced with fresh medium supplemented with $10 \%$ Cell Counting Kit-8 (CCK-8) reagent. Cells were then incubated for an additional $4 \mathrm{~h}$. Finally, the optical density value of each well was determined at $450 \mathrm{~nm}$ using a microplate reader (Bio-Rad Laboratories, Inc.). Each experiment was carried out $\geq 3$ times.

Colony formation assay. Transfected NSCLC cells were seeded into 12 -well plates (500 cells/well) and incubated for 14 days. Subsequently, cells were fixed with $4 \%$ paraformaldehyde for $15 \mathrm{~min}$, stained with $0.1 \%$ crystal violet, and then photographed under a microscope (Leica Microsystems $\mathrm{GmbH})$. Only colonies with $>50$ cells were counted.

Transwell assay. A total of $1 \times 10^{5}$ cells were harvested, resuspended in $100 \mu \mathrm{l}$ serum-free medium and plated into the upper chambers of 24-well Transwell plates (8.0- $\mu \mathrm{m}$ pore size; Corning, Inc.). The lower chamber was supplemented with $600 \mu \mathrm{l}$ medium containing $10 \%$ FBS. Following incubation for $24 \mathrm{~h}$, the medium was removed, and the chamber was washed twice with PBS. Cells on the upper chamber were removed using cotton swabs, while those on the lower surface of the chamber were fixed with $4 \%$ paraformaldehyde for $15 \mathrm{~min}$, stained with $0.1 \%$ crystal violet for $5 \mathrm{~min}$, and then observed under a light microscope (Leica Microsystems $\mathrm{GmbH}$ ).

Luciferase assay. TargetScan (http://www.targetscan.org/) and MIRDB (http://mirdb.org/) databases were used to predict whether miR-564 could directly target plexin A4. The 3'-untranslated region (3'UTR) of plexin A4 was synthesized by Shanghai GenePharma Co., Ltd. and subcloned into a pmirGLO vector (Promega Corporation). Subsequently, A549 and H460 cells were co-transfected with miR-564 mimics or mimics control and plasmids encompassing the wild-type (wt) or mutant (mut) plexin A4-3'UTR using Lipofectamine 3000 (Thermo Fisher Scientific, Inc.).

Western blot analysis. Cells were lysed with RIPA lysis buffer (Beyotime Institute of Biotechnology), and the protein concentration was determined with the NanoDrop 2000 system (Thermo Fisher Scientific, Inc.). A total of $40 \mu \mathrm{g}$ protein was separated by $10 \%$ SDS-PAGE and then electrotransferred onto polyvinylidene difluoride (PVDF) membranes. Following blocking with $5 \%$ milk for $60 \mathrm{~min}$ at room temperature, the membranes were incubated with specific primary antibodies against plexin $\mathrm{A} 4$ and GAPDH at $4^{\circ} \mathrm{C}$ overnight. Subsequently, membranes were incubated with the corresponding secondary antibodies at room temperature for $\sim 2 \mathrm{~h}$. Finally, the blots were visualized using an ECL kit (Beyotime Institute of Biotechnology).

Nude mice xenograft model. Male BALB/c nude mice (6-weeks-old) were obtained from the Vital River Laboratory Animal Technology Company (Beijing, China). The mice were housed in specific pathogen free (SPF) facilities under a $12 \mathrm{~h}$ light/dark cycle, and temperature- and humidity-controlled conditions. Nude mice were subcutaneously injected with $5 \times 10^{6}$ A549 cells. Following 7 days, the tumors were injected with 

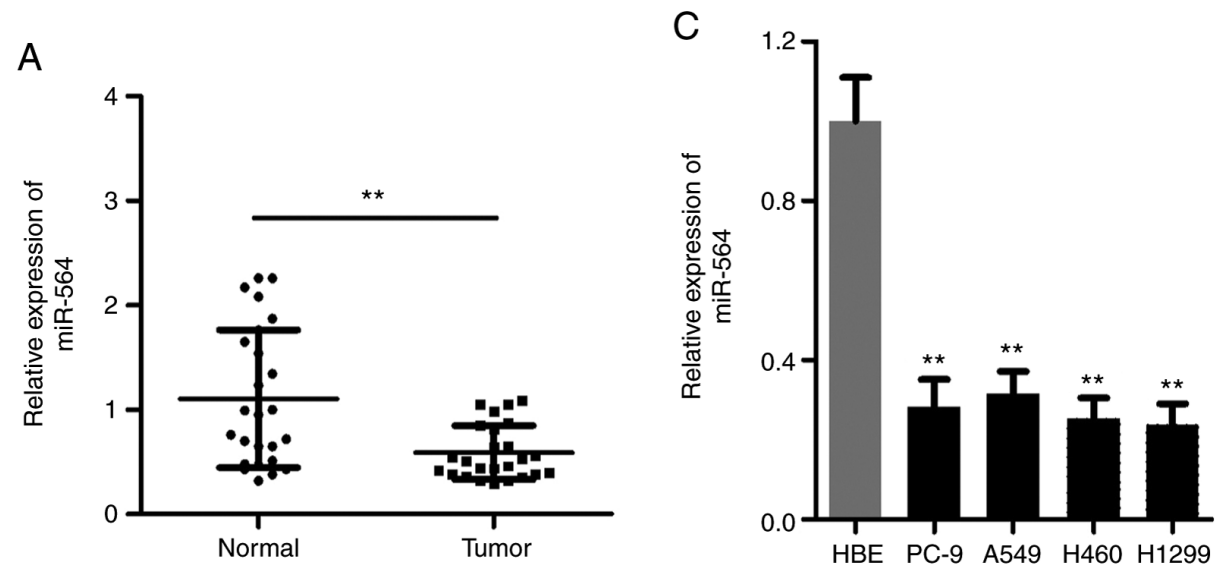

B

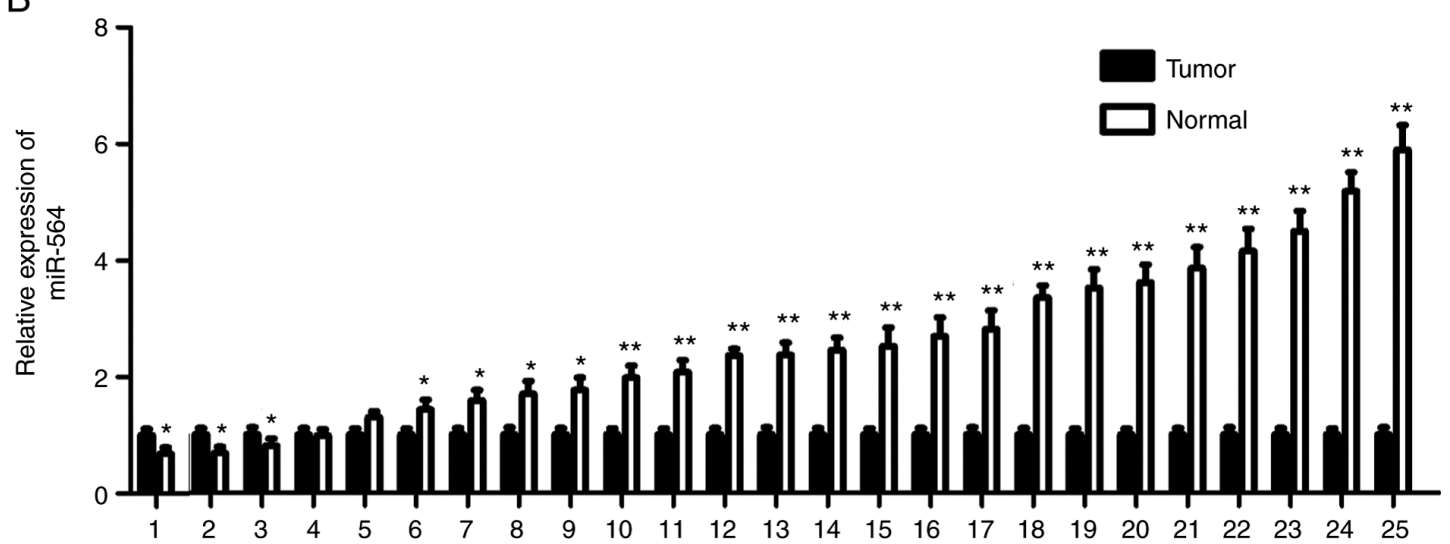

Figure 1. Expression level of miR-564 is downregulated in NSCLC cell lines and tissues. (A and B) miR-564 expression in 25 paired NSCLC tissues compared with the normal tissues was detected using RT-qPCR analysis. (C) MiR-564 expression level in HBE and NSCLC cell lines were examined using RT-qPCR. ${ }^{*} \mathrm{P}<0.05 ;{ }^{* *} \mathrm{P}<0.01$ vs. normal tissue or HBE cells. miR, microRNA; NSCLC, non-small cell lung cancer; RT-qPCR, reverse transcription-quantitative PCR.

$3 \mathrm{nmol}$ of antagomiR-564 or agomir-564 (RiboBio Co., Ltd.) as well as their negative controls respectively. The injections were carried out every 2 days for a period of 20 days. The tumor size (width and length) was measured every 2 days, and tumor volume was calculated using the following formula: Tumor volume $=1 / 2 \mathrm{x}$ (width) ${ }^{2} \mathrm{x}$ length. The animal experiments were approved by the Ethics Committee of the Huaian First People's Hospital. At the end, the mouse were euthanized by intraperitoneal injection of $4 \%$ pentobarbital $(180 \mathrm{mg} / \mathrm{kg})$. Then, the tumors were removed.

Immunohistochemistry (IHC). Paraffin-embedded tumor tissues were cut into $4-\mu \mathrm{m}$ thick sections. Then, the sections were deparaffinized in xylene and rehydrated. Antigen retrieval was performed in a pressure cooker for $30 \mathrm{~min}$ with $10 \mathrm{mM}$ citrate buffer and the activity of endogenous peroxidase was blocked using $0.5 \%$ hydrogen peroxide. Subsequently, the sections were incubated with primary antibodies against Ki67 or plexin $\mathrm{A} 4$ overnight at $4^{\circ} \mathrm{C}$. Immunostaining was carried out using a diaminobenzidine (DAB) staining kit according to the manufacturer's instructions. The stained slides were visualized under a microscope.

Statistical analysis. All statistical analyses were performed with SPSS 20.0 software (IBM Corp.). Data are expressed as the mean \pm standard deviation (SD). Paired and unpaired Student's t-tests were used to evaluate the differences in gene expression between two groups. One-way analysis of variance (ANOVA) with Tukey's test were used to analyze differences between two and multiple groups. $\mathrm{P}<0.05$ was considered to indicate a statistically significant difference.

\section{Results}

miR-564 expression is downregulated in NSCLC tissues and cell lines. RT-qPCR analysis was used to detect the expression levels of miR-564 in 25 paired NSCLC tissues and cell lines. The expression levels of miR-564 were notably reduced in NSCLC tissues compared with those in the matched normal tissues (Fig. 1A and B). Consistent with the previous finding, miR-564 was downregulated in all four NSCLC cell lines (A549, H460, PC-9, and H1200), to differing degrees, compared with the HBE group (Fig. 1C). These results indicated that miR-564 could promote NSCLC development.

miR-564 inhibits A549 and H460 cell proliferation and migration. A549 and H460 cells were transfected with miR-564 mimics, inhibitor or their corresponding negative controls and RT-qPCR analysis was carried out. The results showed that the cell transfection with miR-564 mimics or inhibitor notably increased and decreased the miR-564 expression levels, respectively (Fig. 2A). Furthermore, the CCK- 8 and colony formation assays demonstrated that miR-564 mimics inhibited, while the miR-564 inhibitor 
A
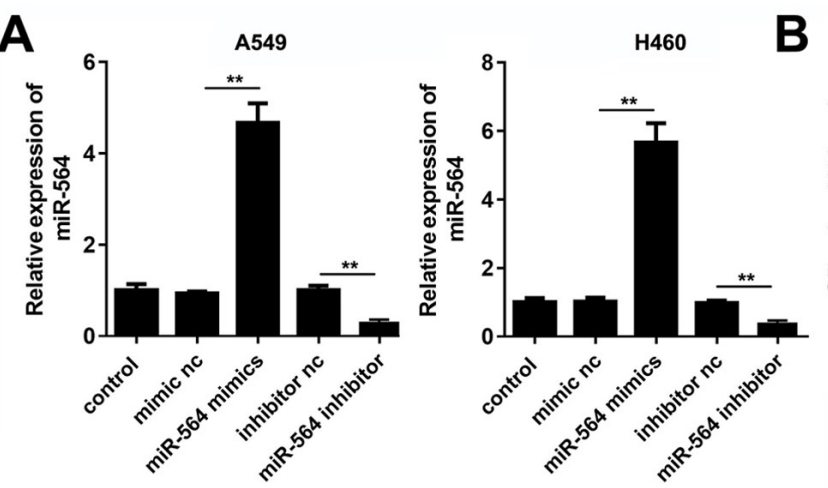

C
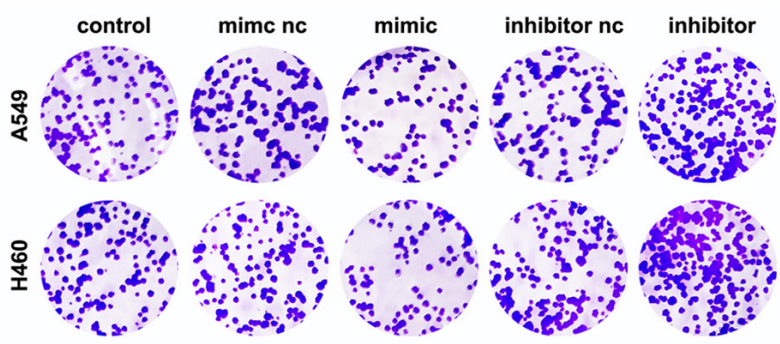

D

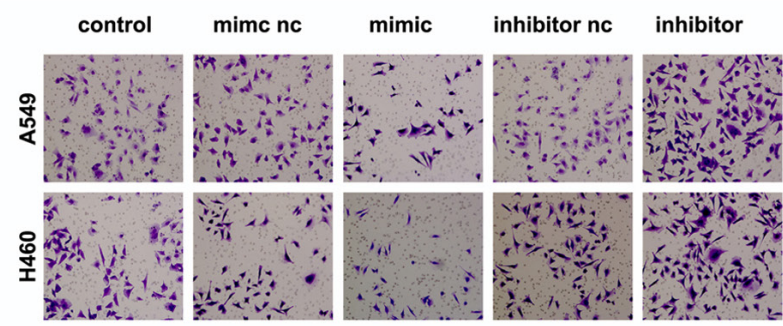

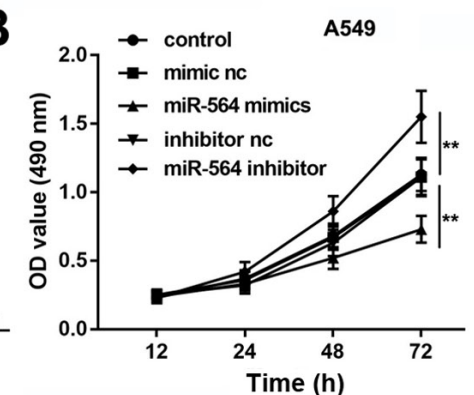
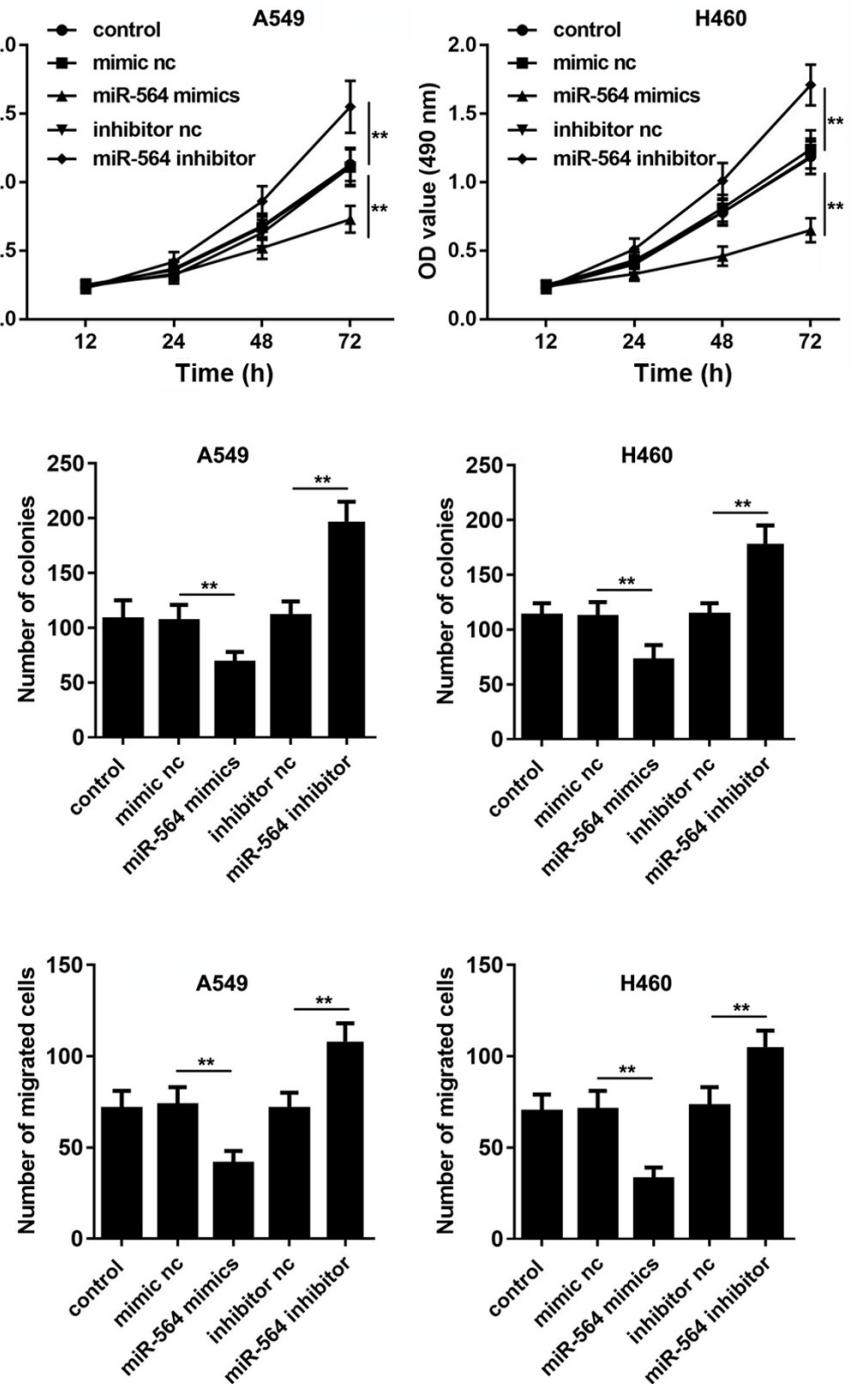

Figure 2. Proliferation and migration of NSCLC cells are inhibited by miR-564 in vitro. miR-564 mimics, miR-564 inhibitor, mimics control or inhibitor control were used to transfect A549 and H460 cells. (A) The expression level of miR-564 after transfection was evaluated using reverse transcription-quantitative PCR. (B) Cell Counting Kit-8 and (C) colony formation assays were performed to evaluate the proliferation of NSCLC cells. (D) Transwell assay was performed to detect cell migration in each group. ${ }^{* *} \mathrm{P}<0.01$. miR, microRNA; NSCLC, non-small cell lung cancer; nc, negative control.

promoted, NSCLC cell viability (Fig. 2B and C). Similarly, the Transwell assays revealed that the cell migration ability was attenuated in A549 and H460 cells following the transfection with miR-564 mimics. By contrast, the cell transfection with miR-564 inhibitor induced the A549 and H460 cell migratory ability (Fig. 2D). Overall, these findings suggested that miR-564 could attenuate NSCLC cell proliferation and migration.

miR-564 directly targets plexin A4 in NSCLC cells. To determine the mechanisms underlying the effects of miR-564 in NSCLC, the mRNA targets of miR-564 were identified using TargetScan and MiRDB databases. A total of 86 genes were predicted as potential targets of miR-564 using both softwares (Fig. 3A). The results of the Gene Ontology (GO) enrichment analysis are shown in Fig. 3B. Among the 86 potential target genes, plexin A4 was selected for subsequent experiments, since it was predicted to be involved in the regulation of cell migration. The predicted binding site between miR-564 and plexin A4 is presented in Fig. 3C. Subsequently, a luciferase reporter assay was performed. The results demonstrated that miR-564 overexpression notably reduced the activity of the reporter plasmid carrying plexin A4-3'-UTR-wt but not that of plexin A4-3'UTR-mut (Fig. 3D). In addition, miR-564 expression negatively regulated the mRNA and protein expression levels of plexin A4 in A549 and H460 cells (Fig. 3E and F). The aforementioned findings suggested that plexin A4 was a direct target of miR-564 in A549 and H460 NSCLC cells.

Plexin A4 overexpression reverses the effect of miR-564 on NSCLC cells. To verify whether plexin A4 could affect the function of miR-564 on NSCLC cell proliferation and migration, A549 and H460 cells were co-transfected with a plexin A4 overexpression plasmid and miR-564 mimics. Then, the expression of plexin A4 was determined by RT-qPCR. The results showed that transfection with a plexin A4 overexpression plasmid significantly elevated the expression level of plexin A4 (Fig. S1). In addition, miR-564 overexpression notably reduced the expression levels of plexin A4, however, its expression levels were restored following transfection with a plexin A4 
A

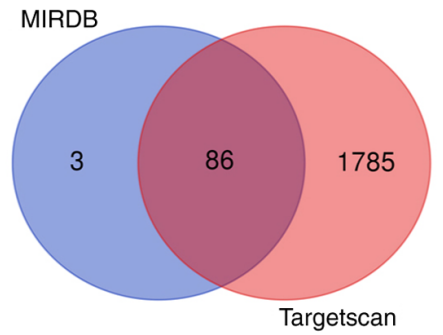

C

Plexin A4 WT5' GGGGGACACACCAAGCCGUGCCU 3'

| || || | |

has-miR-564 $33^{\prime}$ CGGACGACUGUGGCACGGA 5' I $\quad$ ।

Plexin A4 mut5' GGGGGACACACCAAGCGCACGGU3'

B Positive regulation of excitatory postsynaptic... Go analysis Regulation of dendrite morphogenesis Phototransduction, visible light Commissural neuron axon guidance Anterior commissure morphogenesis Canonical Wnt signaling pathway Regulation of cell migration $\longrightarrow$ Plexin A4 Synapse assembly
of insulin secretion

Positive regulation of insulin secretion
Il GTPase mediated signal transduction Cell differentiation

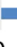
2
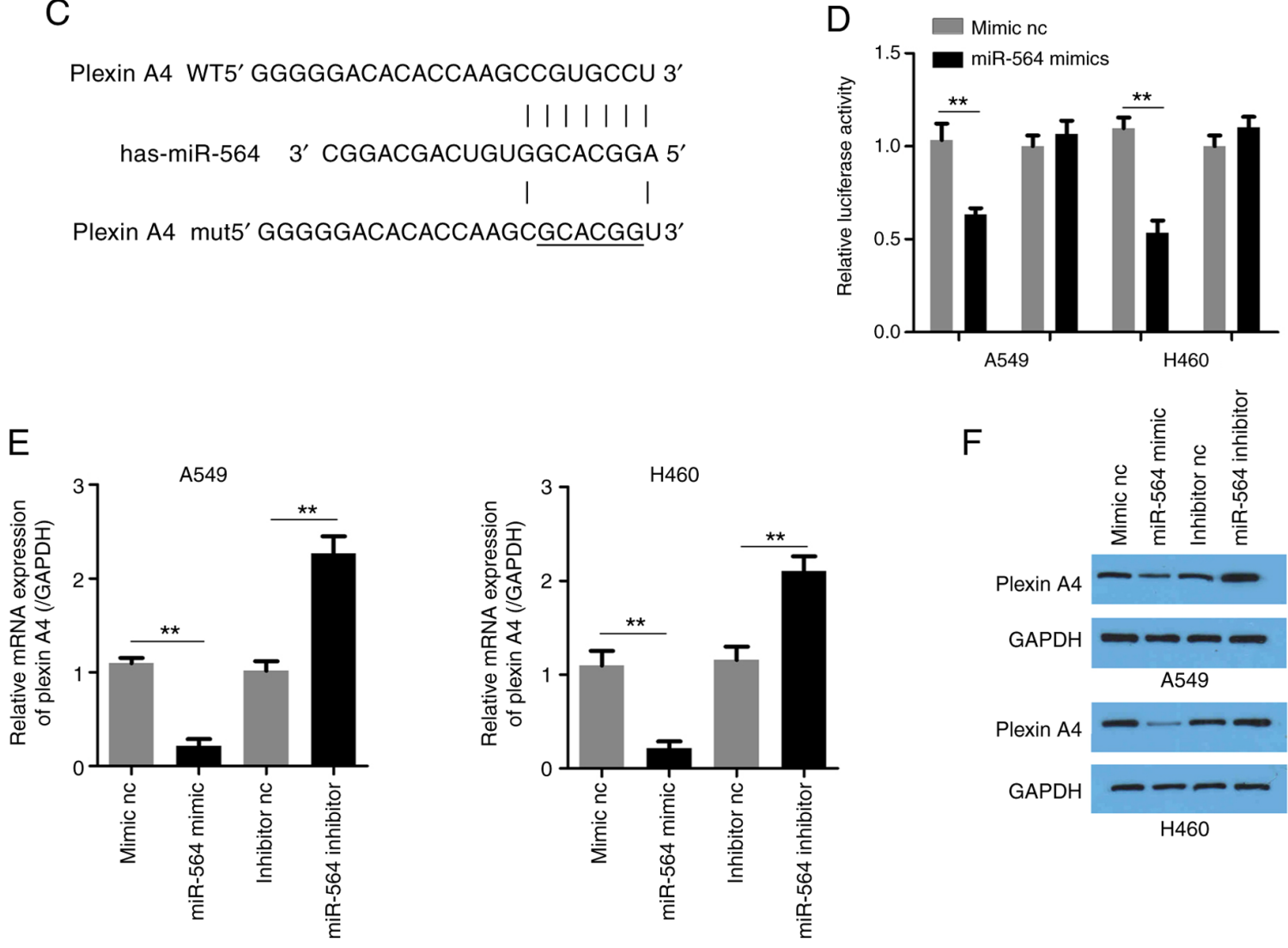

$\mathrm{F}$

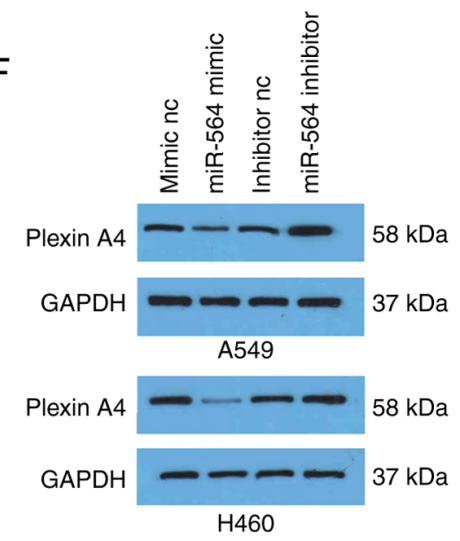

Figure 3. Plexin A4 is a direct target of miR-564 in NSCLC cells. (A) Targetscan 7.2 and MIRDB software were used to predict the targets of miR-564. (B) GO enrichment analysis was performed using the 86 potential target genes. (C) The putative and mutant binding sequences between miR-564 and plexin A4 are presented. (D) The luciferase reporter assay was performed to verify the binding between miR-564 and plexin A4. (E) The mRNA expression of plexin A4 in NSCLC cells was assessed using reverse transcription-quantitative PCR. (F) The relative protein expression level of plexin A4 was assessed via western blot analysis. ${ }^{* *} \mathrm{P}<0.01$. miR, microRNA; NSCLC, non-small cell lung cancer; nc, negative control; go, gene ontology; mut, mutant; WT, wild-type.

overexpression plasmid (Fig. 4A). Furthermore, the CCK-8 and colony formation assays revealed that cell co-transfection with miR-564 mimics and pcDNA3.1-plexin A4 reversed the effect of miR-564 on NSCLC cell proliferation (Fig. 4B and C). Additionally, the overexpression of plexin A4 also reversed the effect of miR-564 on NSCLC cell migration (Fig. 4D). These findings indicated that miR-564 could inhibit the proliferative and migratory ability of NSCLC cells via targeting plexin A4.

miR-564 attenuates tumor growth in mice. The antagomir-564 and agomir-564 were used to inhibit or overexpress the expression of miR-564 in the tumor tissues, respectively. MiR-564 overexpression notably inhibited while miR-564 knockdown promoted the growth of NSCLC cells in vivo (Fig. 5A and B). Furthermore, the tumor weight in the miR-564 silenced group was notably increased compared with the control group while
miR-564 overexpression reduced the tumor weight (Fig. 5C). The largest tumor diameter is $12.8 \mathrm{~mm}$. In addition, the IHC results revealed that the expression of Ki67 and plexin A4 was upregulated after miR-564 silencing while downregulated by miR-564 overexpression (Fig. 5D). To further verify whether the antogomir-564 could inhibit miR-564, the expression of miR-564 was determined in tumor tissues. The results indicated that antogomir-564 could notably attenuate the expression levels of miR-564 while agomir-564 promoted that (Fig. 5E).

\section{Discussion}

In the current study, the expression of miR-564 was significantly decreased in both NSCLC tissues and cell lines. To elucidate the function of miR-564, A549 and H460 cells were transfected with miR-564 mimics, to increase its expression, 

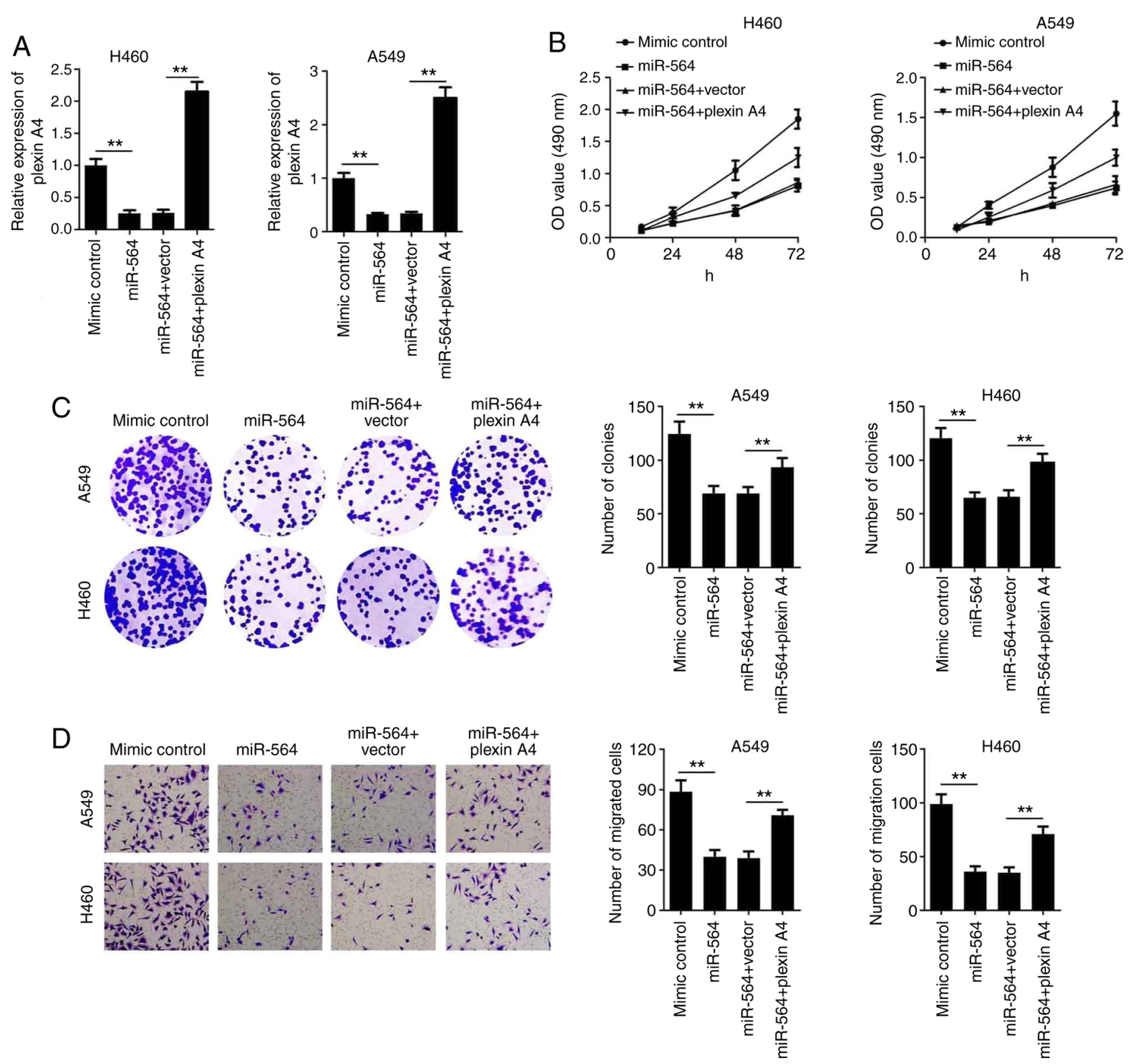

Figure 4. Overexpression of plexin A4 reverses the effect of miR-564 on proliferation and migration of NSCLC cells. (A) Reverse transcription-quantitative PCR was performed to evaluate the plexin A4 expression. Proliferative ability of NSCLC cells in each group was measured by (B) Cell Counting Kit-8 and (C) colony formation assays. (D) In order to detect cell migration, Transwell assays were performed. ${ }^{* *} \mathrm{P}<0.01$. miR, microRNA; NSCLC, non-small cell lung cancer.

or with miR-564 inhibitor to downregulate its endogenous expression. The functional studies revealed the inhibitory effect of miR-564 on NSCLC cell proliferation and migration. Mechanistically, miR-564 directly targeted plexin A4 to inhibit its expression.

It has been reported that miR-564 exerts an antitumor effect in several types of cancer, including NSCLC. More specifically, miR-564 is downregulated in NSCLC (20). Herein, the A549 and H460 NSCLC cell lines were used for the in vitro experiments, which was inconsistent with a previous study, where A549 and H1299 cell lines were utilized. In addition, the study assessed the effect of ectopic miR-564 expression on tumor growth in vivo. In the present study, the role of miR-564 knockdown on NSCLC growth was investigated in vivo via injecting the miR-564 antagomiR into mice. Antagomirs and agomirs have been widely used in vitro and in vivo to knockdown target genes $(21,22)$. The results demonstrated that the miR-564 antagomiR significantly downregulated miR-564 in tumor tissues. Furthermore, miR-564 knockdown notably enhanced NSCLC cell growth. Therefore, the current findings further confirmed the antitumor effect of miR-564 in NSCLC. However, in the present study, the tumor size reached about $30 \mathrm{~mm} 3$ when the treatment was performed. In the future study, we will perform the administration of antagomir-564 or agomir-564 when the tumors reach a higher volume to check if miR-564 can still change the growth of NSCLC cells.

miRNAs can modulate the expression of their target genes via binding to specific regions $(23,24)$. It has been reported that miR-564 directly targets astrocyte-elevated gene-1 (AEG1), transforming growth factor (TGF)- $\beta 1$ and E2F transcription factor 3 (E2F3) $(12,25,26)$. Herein, the possible targets of miR-564 were accurately predicted using TargetScan and MirDB software. Among the possible target genes, plexin A4 
A
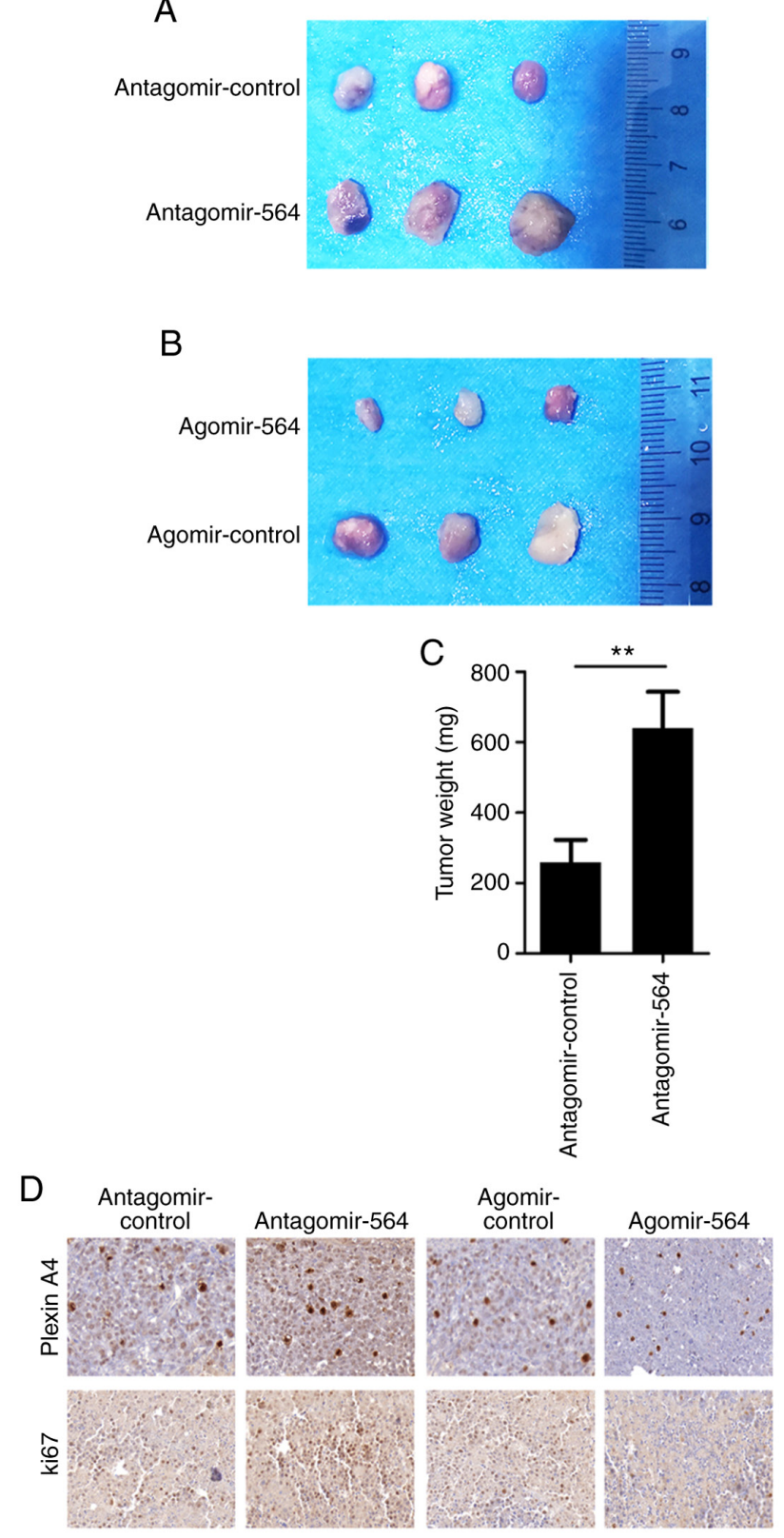
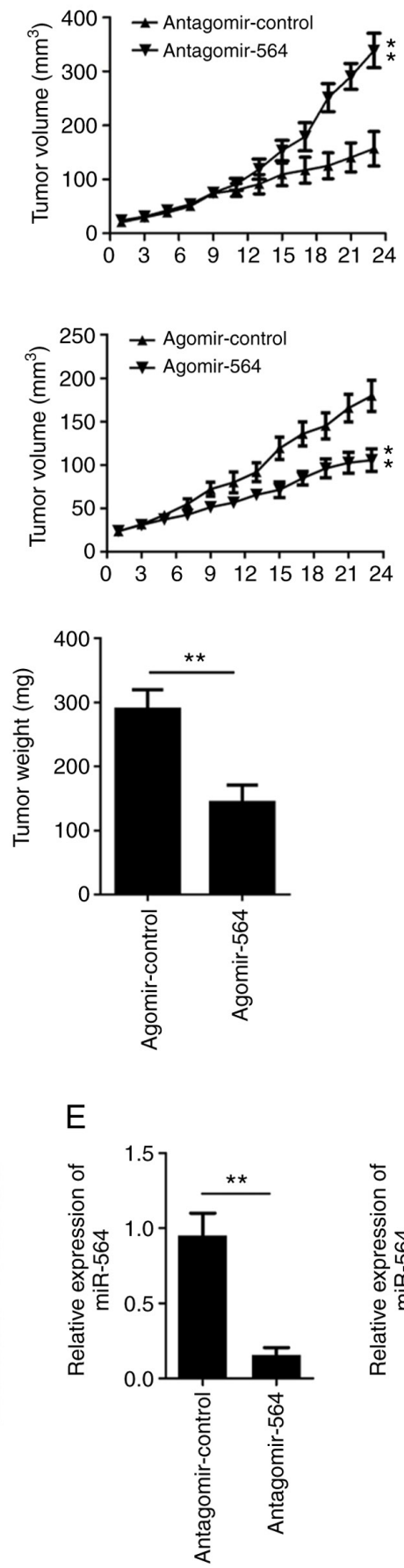

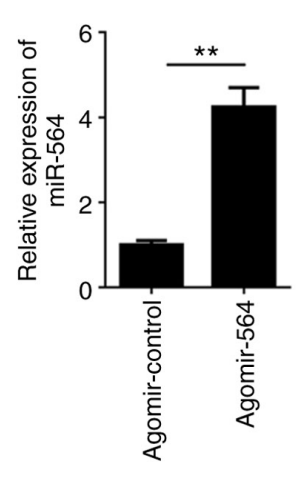

Figure 5. miR-564 silencing promotes NSCLC tumor growth in vivo. (A) Image and growth curve of tumors in antagomir-control and antagomir-564 groups. (B) Image and growth curve of tumors in agomir-control and agomir-564 groups. (C) Weight of tumors in each group. (D) Immunohistochemical staining of Ki67 and plexin A4 in tumor tissue. (E) RT-qPCR was used to evaluate the expression level of miR-564 in the tumor tissues. ${ }^{* *} \mathrm{P}<0.01$. miR, microRNA; NSCLC, non-small cell lung cancer.

was selected for further experiments, since it was predicted to be involved in cell migration.

Except from class 3 semaphorins (Semas) such as Sema3a, which binds to both neuropilin (Nrp)-1 and Nrp-2, plexins are receptors of Semas. Sema3a signaling is mediated through plexin A4, as well as other plexin A proteins $(27,28)$. In addition, the Sema3a/Nrp-1/plexinA4 signaling pathway is involved in the inflammatory immune response and tumor cell growth (29). In the present study, the luciferase reporter assay and rescue experiments predicted and verified that miR-564 could directly target plexin A4 in NSCLC cells.
It has been reported that plexin A4 modulates the phosphorylation status of phosphatase and tensin homolog (PTEN) (29). Therefore, the effect of miR-564 on PTEN phosphorylation will be further investigated in future studies.

In conclusion, the current study showed that miR-564 was downregulated in NSCLC. Furthermore, miR-564 overexpression attenuated NSCLC proliferation and migration via upregulating plexin A4 in vitro and in vivo. Therefore, miR-564 and plexin A4 could be considered as potential therapeutic targets for NSCLC. 


\section{Acknowledgements}

Not applicable.

\section{Funding}

Not applicable.

\section{Availability of data and materials}

The datasets used and/or analyzed during the current study are available from the corresponding author on reasonable request.

\section{Authors' contributions}

HD performed the in vitro study. BG performed the in vivo study and the histological examination. LL analyzed and interpreted the patient data. YN contributed to the statistical analysis. SC designed the study and wrote the manuscript and agreed to be accountable for all aspects of the work in ensuring that questions related to the accuracy or integrity of any part of the work are appropriately investigated and resolved. HD, BG and SC confirmed the authenticity of the raw data. All authors read and approved the final manuscript.

\section{Ethics approval and consent to participate}

The current study was approved by the Ethics Committee of the Huaian First People's Hospital and was performed in accordance with the Declaration of Helsinki. Patients signed informed consent in order for their specimens to be used for scientific research.

\section{Patient consent for publication}

Not Applicable.

\section{Competing interests}

The authors declare that they have no competing interests.

\section{References}

1. Zhang X, Xiong Y, Xia Q, Wu F, Liu L, Zhou Y, Zeng L, Zhou C Xia C, Jiang W, et al: Efficacy and safety of apatinib plus vinorelbine in patients with wild-type advanced non-small cell lung cancer after second-line treatment failure: A nonrandomized clinical trial. JAMA Netw Open 3: e201226, 2020.

2. Innos K, Oselin K, Laisaar T and Aareleid T: Patterns of survival and surgical treatment in lung cancer patients in Estonia by histologic type and stage, 1996-2016. Acta Oncol 58: 1549-1556, 2019.

3. Zare A, Ganji M, Omrani MD, Alipoor B and Ghaedi H: Gastric cancer MicroRNAs Meta-signature. Int J Mol Cell Med 8: 94-102, 2019.

4. Mohr AM and Mott JL: Overview of microRNA biology. Semin Liver Dis 35: 3-11, 2015.

5. Jafari $\mathrm{N}$ and Abediankenari S: MicroRNA-34 dysregulation in gastric cancer and gastric cancer stem cell. Tumour Biol 39: $1010428317701652,2017$.

6. Kanchan RK, Siddiqui JA, Mahapatra S, Batra SK and Nasser MW: microRNAs orchestrate pathophysiology of breast cancer brain metastasis: Advances in therapy. Mol Cancer 19: 29, 2020.

7. Gon Y, Shimizu T, Mizumura K, Maruoka S and Hikichi M: Molecular techniques for respiratory diseases: MicroRNA and extracellular vesicles. Respirology 25: 149-160, 2020.

8. Liang C, Xu Y, Ge H, Xing B, Li G, Li G and Wu J: miR-564 inhibits hepatocellular carcinoma cell proliferation and invasion by targeting the GRB2-ERK1/2-AKT axis. Oncotarget 8: 107543-107557, 2017.
9. Ru N, Zhang F, Liang J, Du Y, Wu W, Wang F and Liu X: MiR-564 is down-regulated in osteosarcoma and inhibits the proliferation of osteosarcoma cells via targeting Akt. Gene 645: 163-169, 2018.

10. Meng FJ, Meng FM, Wu HX and Cao XF: miR-564 inhibited metastasis and proliferation of prostate cancer by targeting MLLT3. Eur Rev Med Pharmacol Sci 21: 4828-4834, 2017.

11. Guo Y, Qi Y, Guo A, Du C, Zhang R and Chu X: miR-564 is downregulated in gastric carcinoma and targets E2F3. Oncol Lett 13: 4155-4160, 2017.

12. Jiang C, Shen F, Du J, Hu Z, Li X, Su J, Wang X and Huang X: MicroRNA-564 is downregulated in glioblastoma and inhibited proliferation and invasion of glioblastoma cells by targeting TGF- 31 . Oncotarget 7: 56200-56208, 2016.

13. Tran TS, Kolodkin AL and Bharadwaj R: Semaphorin regulation of cellular morphology. Annu Rev Cell Dev Biol 23: 263-292, 2007.

14. Matsuoka RL, Nguyen-Ba-Charvet KT, Parray A, Badea TC, Chedotal A and Kolodkin AL: Transmembrane semaphorin signalling controls laminar stratification in the mammalian retina. Nature 470: 259-263, 2011.

15. Mauti O, Domaniskaya E, Andermatt I, Sadhu R and Stoeckli ET: Semaphorin6A acts as a gate keeper between the central and the peripheral nervous system. Neural Dev 2: 28, 2007.

16. Catalano A, Lazzarini R, Di Nuzzo S, Orciari S and Procopio A: The plexin-A1 receptor activates vascular endothelial growth factor-receptor 2 and nuclear factor-kappaB to mediate survival and anchorage-independent growth of malignant mesothelioma cells. Cancer Res 69: 1485-1493, 2009.

17. Yamamoto M, Suzuki K, Okuno T, Ogata T, Takegahara N, Takamatsu H, Mizui M, Taniguchi M, Chédotal A, Suto F, et al: Plexin-A4 negatively regulates $\mathrm{T}$ lymphocyte responses. Int Immunol 20: 413-420, 2008.

18. Kigel B, Rabinowicz N, Varshavsky A, Kessler O and Neufeld G: Plexin-A4 promotes tumor progression and tumor angiogenesis by enhancement of VEGF and bFGF signaling. Blood 118: 4285-4296, 2011.

19. Livak KJ and Schmittgen TD: Analysis of relative gene expression data using real-time quantitative PCR and the 2(-Delta Delta C(T)) method. Methods 25: 402-408, 2001.

20. Yang B, Jia L, Guo Q, Ren H, Hu D, Zhou X, Ren Q, Hu Y and Xie T: MiR-564 functions as a tumor suppressor in human lung cancer by targeting ZIC3. Biochem Biophys Res Commun 467: 690-966, 2015.

21. Wang H, Chao K, Ng SC, Bai AH, Yu Q, Yu J, Li M, Cui Y, Chen M, Hu JF and Zhang S: Pro-inflammatory miR-223 mediates the cross-talk between the IL23 pathway and the intestinal barrier in inflammatory bowel disease. Genome Biol 17: 58, 2016.

22. Wu Y, Heinrichs J, Bastian D, Fu J, Nguyen H, Schutt S, Liu Y, Jin J, Liu C, Li QJ, et al: MicroRNA-17-92 controls T-cell responses in graft-versus-host disease and leukemia relapse in mice. Blood 126: 1314-1323, 2015.

23. Whipple AJ, Breton-Provencher V, Jacobs HN, Chitta UK, Sur M and Sharp PA: Imprinted maternally expressed microRNAs antagonize paternally driven gene programs in neurons. Mol Cell 78: 85-95.e8, 2020.

24. Basak A, Munschauer M, Lareau CA, Montbleau KE, Ulirsch JC, Hartigan CR, Schenone M, Lian J, Wang Y, Huang Y, et al: Control of human hemoglobin switching by LIN28B-mediated regulation of BCL11A translation. Nat Genet 52: 138-145, 2020.

25. Xiao L, Tang T, Huang Y and Guo J: MiR-564 promotes hypertrophic scar formation through TGF- $\beta 1$ upregulation. G Ital Dermatol Venereol 154: 186-191, 2019.

26. Song Z, Yang H, Wu X, Kong $\mathrm{C}$ and $\mathrm{Xu} C$ : microRNA-564 inhibits the aggressive phenotypes of papillary thyroid cancer by directly targeting astrocyte-elevated gene-1. Onco Targets Ther 12: 4869-4881, 2019.

27. Casazza A, Laoui D, Wenes M, Rizzolio S, Bassani N, Mambretti M, Deschoemaeker S, Van Ginderachter JA, Tamagnone L and Mazzone M: Impeding macrophage entry into hypoxic tumor areas by Sema3A/Nrp1 signaling blockade inhibits angiogenesis and restores antitumor immunity. Cancer Cell 24: 695-709, 2013.

28. He Z and Tessier-Lavigne M: Neuropilin is a receptor for the axonal chemorepellent Semaphorin III. Cell 90: 739-751, 1997.

29. PodojilJR, Chiang MY,IferganI,CopelandR,Liu LN,MalovesteS, Langermann S, Liebenson D, Balabanov R, Chi H, et al: B7-H4 modulates regulatory $\mathrm{CD} 4(+) \mathrm{T}$ Cell induction and function via ligation of a semaphorin 3a/Plexin A4/Neuropilin-1 complex. J Immunol 201: 897-907, 2018.

(i) (9) This work is licensed under a Creative Commons Attribution-NonCommercial-NoDerivatives 4.0 International (CC BY-NC-ND 4.0) License. 